Article

\title{
Lévy Processes Linked to the Lower-Incomplete Gamma Function
}

\author{
Luisa Beghin * and Costantino Ricciuti
}

Department of Statistical Science, Sapienza University of Rome, P.le A. Moro 5, 00185 Roma, Italy; costantino.ricciuti@uniroma1.it

* Correspondence: luisa.beghin@uniroma1.it

\begin{abstract}
We start by defining a subordinator by means of the lower-incomplete gamma function. This can be considered as an approximation of the stable subordinator, easier to be handled in view of its finite activity. A tempered version is also considered in order to overcome the drawback of infinite moments. Then, we study Lévy processes that are time-changed by these subordinators with particular attention to the Brownian case. An approximation of the fractional derivative (as well as of the fractional power of operators) arises from the analysis of governing equations. Finally, we show that time-changing the fractional Brownian motion produces a model of anomalous diffusion, which exhibits a sub-diffusive behavior.
\end{abstract}

Keywords: incomplete-gamma function; anomalous diffusions; Lévy processes; subordination; fractional operators

AMS Mathematical Subject Classification: 33B20; 26A33; 60G51; 60J65; 34A08

Citation: Beghin, L.; Ricciuti, C. Lévy Processes Linked to the Lower-Incomplete Gamma Function. Fractal Fract. 2021, 5, 72. https:// doi.org/10.3390/fractalfract5030072

Academic Editors: Mirko D'Ovidio and Sergei Fedotov

Received: 22 June 2021

Accepted: 13 July 2021

Published: 17 July 2021

Publisher's Note: MDPI stays neutral with regard to jurisdictional claims in published maps and institutional affiliations.

Copyright: (c) 2021 by the authors. Licensee MDPI, Basel, Switzerland. This article is an open access article distributed under the terms and conditions of the Creative Commons Attribution (CC BY) license (https:// creativecommons.org/licenses/by/ $4.0 /)$.

\section{Introduction}

In the spirit of [1], we consider here a subordinator $S_{\alpha}(t), t \geq 0$, defined by means of the lower-incomplete gamma function of parameter $\alpha \in(0,1]$, i.e.,

$$
\gamma(\alpha, x)=\int_{0}^{x} e^{-w} w^{\alpha-1} d w, \quad x>0 .
$$

More precisely, we define $S_{\alpha}(t), t \geq 0$, as a non-decreasing Lévy process with Laplace exponent $\alpha \gamma(\alpha ; \eta)$. We will see that, in the special case $\alpha=1$, it reduces to a homogeneous Poisson process, while, in general, it can be represented as a compound Poisson process with positive jumps in size greater than one. Such a process retains many properties of the stable subordinator, e.g., the tail behavior of the distribution and the asymptotic form of the fractional moments, even if it loses the property of self-similarity. A standard reference for the theory of stable processes is [2].

By a slight modification, we are led to a new subordinator whose jumps are greater than $\epsilon>0$, which converges to a stable one in the limit for $\epsilon \rightarrow 0$. We prove that its density $q_{\epsilon}(x, t)$ solves an equation where a perturbation of the Riemann fractional derivative appears. When $\epsilon \rightarrow 0$, such an operator reduces to the Riemann derivative, and we obtain the well known equation governing the stable density. For an introduction to fractional derivatives and fractional equations consult [3].

The above framework can be extended to the so-called multivariate subordinators, i.e., multidimensional Lévy processes with increasing marginal components (for their properties and applications see e.g., [4,5]).

In order to overcome the drawback of infinite moments of $S_{\alpha}$, we consider a tempered version of our subordinator, say $S_{\alpha, \theta}(t), t \geq 0$, where $\theta \geq 0$ is the tempering parameter, whose distribution displays finite moments of any integer order. 
We use these subordinators as independent random times of well-known Lévy processes. As for other subordinated processes already studied in the literature, the timechange allows us to maintain certain properties of the external process and to simultaneously modify other features (see [6] for the general theory).

When considering the process $B\left(S_{\alpha, \theta}(t)\right), t \geq 0$, where $B:=\{B(t), t \geq 0\}$ is a standard Brownian motion and $S_{\alpha, \theta}$ is supposed as independent from $B$, we obtain the following auto-covariance function

$$
\operatorname{Cov}\left(B\left(S_{\alpha, \theta}(t)\right), B\left(S_{\alpha, \theta}(\tau)\right)\right)=\alpha(t \wedge \tau) \theta^{\alpha-1} e^{-\theta}, \quad t, \tau \geq 0 .
$$

Even if it is linear in the time argument, as for the standard Brownian motion, the parameters $\alpha$ and $\theta$ model the deviation from the dependence structure of $B$ : in particular, for $\theta \rightarrow 0$ and for $\alpha$ strictly less than 1, the auto-covariance tends to infinity, for any $t$.

Finally, we consider a fractional Brownian motion subordinated by $S_{\alpha}(t)$ (for basic notions on the fractional Brownian motion see e.g., [7]). We show that the model obtained still displays long-range dependence, with a rate depending not only on the Hurst index $H$ but also on $\alpha$. It was proven to behave asymptotically as a subdiffusion, depending on the value of the parameter $\alpha$ : the subdiffusive behavior was more marked the greater the value of $\alpha$ (for any fixed $H$ ). We recall that a process is said to be subdiffusive if, for large times $t$, the mean square displacement grows as $t^{\gamma}$ with $\gamma<1$. We refer to [8] for an overview on anomalous diffusion models and their applications.

\section{Basic Notions and Preliminary Results}

We recall the following definition: a function $\varphi:(0, \infty) \rightarrow \mathbb{R}$ is a Bernstein function if $\varphi$ is of class $C^{\infty}, \varphi(\eta) \geq 0$, for any $\eta>0$, and

$$
(-1)^{n-1} \frac{d^{n}}{d x^{n}} \varphi(\eta) \geq 0,
$$

for any $n \in \mathbb{N}$ and $\eta>0$. It is well known that any Bernstein function $\varphi$ admits the following representation

$$
\varphi(\eta)=a+b \eta+\int_{0}^{+\infty}\left(1-e^{-s \eta}\right) v(d s),
$$

for $a, b \geq 0$ and where $v(\cdot)$ denotes a measure on $(0,+\infty)$ such that

$$
\int_{0}^{+\infty}(s \wedge 1) v(d s)<\infty
$$

The triplet $(a, b, v)$ is called the Lévy triplet of the Bernstein function $\varphi$ (see, for example, [9], p. 21) and $v(\cdot)$ is a Lévy measure.

Finally, a Bernstein function $\varphi$ is complete if and only if its Lévy measure in (3) has a completely monotone density $m(\cdot)$ with respect to the Lebesgue measure, i.e., the following representation holds for a completely monotone function $m(\cdot)$ :

$$
\varphi(\eta)=a+b \eta+\int_{0}^{+\infty}\left(1-e^{-s \eta}\right) m(s) d s .
$$

\subsection{Univariate Subordinators}

We now recall that a subordinator $S(t), t \geq 0$, is a Lévy process with non-decreasing paths and that, for any Bernstein function $\varphi$, there exists a subordinator $S(t)$ such that

$$
\mathbb{E} e^{-\eta S(t)}=e^{-t \varphi(\eta)}
$$


(see, for example, [10,11]). In the special case where $\varphi(\eta)=\eta^{\alpha}$, for $\alpha \in(0,1)$, it is wellknown that $S:=\{S(t), t \geq 0\}$ is a $\alpha$-stable subordinator and its density satisfies the following equation:

$$
\frac{\partial}{\partial t} h(x, t)=-\frac{\partial^{\alpha}}{\partial x^{\alpha}} h(x, t), \quad h(x, 0)=\delta(x),
$$

for $x, t \geq 0$, where $\frac{\partial^{\alpha}}{\partial x^{\alpha}}$ is the Riemann-Liouville fractional derivative of order $\alpha$, defined as

$$
\frac{\partial^{\alpha}}{\partial x^{\alpha}} f(x)=\left\{\begin{array}{l}
\frac{1}{\Gamma(1-\alpha)} \frac{d}{d x} \int_{0}^{x} \frac{f(t)}{(x-t)^{\alpha}} d t, \quad \alpha \in(0,1), \\
\frac{d}{d x} f(x), \quad \alpha=1
\end{array}\right.
$$

for a locally integrable function $f$ on $(0,+\infty)$ (see [12], p. 70). This can be easily checked by considering formula (2.2.36) in [12] and applying the Laplace transform to both members of (5), which gives $\widetilde{h}(\eta, t)=e^{-t \eta^{\alpha}}$.

\subsection{Multivariate Subordinators}

In the multivariate case, we recall that a subordinator in the sense of $[4,5]$ is a $d$ dimensional Lévy process with increasing marginal components. We denote a multivariate subordinator by

$$
\left(S_{1}(t), S_{2}(t), \ldots, S_{d}(t)\right) .
$$

The multivariate Lévy measure $v\left(d x_{1}, \ldots, d x_{d}\right)$ satisfies the following condition

$$
\int_{\mathbb{R}_{+}^{d}} \min \left(1, \sqrt{x_{1}^{2}+\cdots+x_{d}^{2}}\right) v\left(d x_{1}, \ldots, d x_{d}\right)<\infty,
$$

where $\mathbb{R}_{+}^{d}=\left\{\left(x_{1}, \ldots, x_{d}\right) \in \mathbb{R}^{d}: x_{1} \geq 0, x_{2} \geq 0, \ldots, x_{d} \geq 0\right\}$.

Its $d$-dimensional Laplace transform reads

$$
\mathbb{E} e^{-\left(\eta_{1} S_{1}(t)+\eta_{2} S_{2}(t)+\cdots+\eta_{d} S_{d}(t)\right)}=e^{-t \Phi\left(\eta_{1}, \ldots, \eta_{d}\right)}, \quad \eta_{1} \geq 0 \ldots \eta_{d} \geq 0,
$$

where

$$
\Phi\left(\eta_{1}, \ldots, \eta_{d}\right)=\int_{\mathbb{R}_{+}^{d}}\left[1-e^{-\left(\eta_{1} x_{1}+\cdots+\eta_{d} x_{d}\right)}\right] v\left(d x_{1}, \ldots, d x_{d}\right)
$$

is a multivariate Bernstein function.

A $d$-dimensional subordinator is said to be stable if, using the spherical variables $\rho \in(0, \infty)$ and $\theta \in B^{d-1}$ ( $B^{d-1}$ denoting the $d$-1-dimensional unit sphere), its Lévy measure can be expressed as

$$
v(d \rho, d \theta)=C \rho^{-\alpha-1} M(d \theta),
$$

where $M(d \theta)$ is a probability measure on $B_{+}^{d-1}=B^{d-1} \cap R_{+}^{d}$. In other words, a $d$-dimensional stable subordinator is a multivariate stable process with increasing marginal components.

In this case, the Bernstein function reads

$$
\Phi(\eta)=k \int_{B_{+}^{d-1}}(\theta \cdot \eta)^{\alpha} M(d \theta), \quad \eta=\left(\eta_{1}, \ldots, \eta_{d}\right) .
$$

By Laplace inversion, the density $q(x, t), x \in R_{+}^{d}, t \geq 0$ of a multivariate stable subordinator satisfies the following equation

$$
\frac{\partial}{\partial t} q(x, t)=-k \int_{B_{+}^{d-1}}\left(\nabla_{x} \cdot \theta\right)^{\alpha} q(x, t) M(d \theta)
$$

where $\left(\nabla_{x} \cdot \theta\right)^{\alpha}$ is the fractional directional derivative along the unit vector $\theta$, defined as 


$$
\left(\nabla_{x} \cdot \theta\right)^{\alpha} h(x):=k \int_{0}^{\infty}(h(x)-h(x-r \theta)) r^{-\alpha-1} d r .
$$

Thus, the operator on the right-hand side of (6), also studied in $[3,13]$, is the average, under the measure $M(d \theta)$, of $\left(\nabla_{x} \cdot \theta\right)^{\alpha}$. For $d=2$, we have $\theta=(\cos \beta, \sin \beta)$, and the operator takes the following form

$$
-k \int_{0}^{\frac{\pi}{2}}\left(\cos \beta \frac{\partial}{\partial x_{1}}+\sin \beta \frac{\partial}{\partial x_{2}}\right)^{\alpha} q\left(x_{1}, x_{2}, t\right) M(d \beta) .
$$

\subsection{Fractional Equation Satisfied by the Incomplete Gamma Function}

The incomplete Gamma function defined in (1) is a Bernstein function. Indeed it is non-negative, $C^{\infty}$ and null at the origin with derivatives satisfying

$$
\begin{aligned}
\frac{d}{d \eta} \gamma(\alpha ; \eta) & =e^{-\eta} \eta^{\alpha-1} \geq 0 \\
\frac{d^{2}}{d \eta^{2}} \gamma(\alpha ; \eta) & =-\frac{d}{d \eta} \gamma(\alpha ; \eta)+(\alpha-1) e^{-\eta} \eta^{\alpha-2} \leq 0 \\
\frac{d^{3}}{d \eta^{3}} \gamma(\alpha ; \eta) & =-\frac{d^{2}}{d \eta^{2}} \gamma(\alpha ; \eta)-(\alpha-1) e^{-\eta} \eta^{\alpha-2}+(\alpha-1)(\alpha-2) e^{-\eta} \eta^{\alpha-3} \geq 0
\end{aligned}
$$

and so on.

Preliminarily, we show that the lower-incomplete Gamma function (1) solves the following integro-differential equation

$$
\frac{\alpha}{\Gamma(1-\alpha)} \int_{0}^{x} \frac{d}{d s} u(x-s) \Gamma(-\alpha, s) d s=\Gamma(\alpha)-u(x), \quad u(0)=0,
$$

where $\Gamma(\beta, x)=\int_{x}^{\infty} e^{-w} w^{\beta-1} d w$ is the upper incomplete Gamma function (which is defined for any $\beta, x \in \mathbb{R}$ and is real-valued for $x \geq 0$ ). Up to a multiplication by $\alpha$, the operator on the left-side is the Caputo fractional derivative with tempered kernel (see, e.g., [14]). We observe that (7) is a relaxation equation because the solution $u(x)=\gamma(\alpha, x)$ converges to the stationary solution $\tilde{u}(x)=\Gamma(\alpha)$ as $x \rightarrow \infty$.

Let now $u: \mathbb{R}^{+} \rightarrow \mathbb{R}^{+}$be an absolutely continuous function, such that $|u(x)| \leq c e^{k x}$, for some $c, k>0$ and for any $x \geq 0$; then, we define the operator

$$
\mathcal{D}_{t}^{\lambda, \rho} u(t):=\frac{\rho \lambda^{\rho}}{\Gamma(1-\rho)} \int_{0}^{t} \frac{d}{d t} u(t-s) \Gamma(-\rho ; \lambda s) d s, \quad \rho \in(0,1), \lambda>0 .
$$

It was proven in [1] that $f(t)=\Gamma(\rho ; \lambda t)$ is the eigenfunction of the operator $\mathcal{D}_{t}^{\lambda, \rho}$, i.e., that $\mathcal{D}_{t}^{\lambda, \rho} f=-\lambda^{\rho} f$. Then, by recalling that $\Gamma(\alpha ; x)+\gamma(\alpha ; x)=\Gamma(\alpha)$, it is easy to check that the Cauchy problem (7) is satisfied. Indeed, $\mathcal{D}_{t}^{\lambda, \rho} K=1$, for any $K \in \mathbb{R}$, by (8) and, moreover, $\gamma(\alpha ; \cdot)$ is absolutely continuous on $\mathbb{R}^{+}$and $|\gamma(\alpha ; x)| \leq \Gamma(\alpha) \leq \Gamma(\alpha) e^{k x}$, for any $x, k \geq 0$.

As an alternative proof, we recall that the Laplace transform of (8) is given by

$$
\int_{0}^{+\infty} e^{-\theta t} \mathcal{D}_{t}^{\lambda, \rho} u(t) d t=\left[(\theta+\lambda)^{\rho}-\lambda^{\rho}\right] \widetilde{u}(\theta)-\frac{\left[(\theta+\lambda)^{\rho}-\lambda^{\rho}\right]}{\theta} u(0), \quad \theta>0
$$

(see [1]); moreover,

$$
\int_{0}^{+\infty} e^{-\theta x} \gamma(\alpha ; x) d x=\int_{0}^{+\infty} e^{-w} w^{\alpha-1} \int_{w}^{+\infty} e^{-\theta x} d x d w=\frac{\Gamma(\alpha)}{\theta(\theta+1)^{\alpha}},
$$


so that the Laplace transforms of the two sides of (7) coincide. We can easily check that, for $\alpha=1$, the Equation (7) reduces to

$$
\frac{d}{d x} u(x)=1-u(x)
$$

which (for $u(0)=0$ ) is satisfied by $u(x)=1-e^{-x}=\gamma(1 ; x)$, even though the expression of $\mathcal{D}_{t}^{\lambda, \rho}$ given in (8) is not well-defined in this special case.

\section{The Subordinator $S_{\alpha}$}

\subsection{Definition and Properties}

We start by considering the subordinator defined by means of the lower-incomplete gamma function, i.e., with Laplace exponent $\alpha \gamma(\alpha ; \eta)$, for $\alpha \in(0,1]$.

Theorem 1. Let $\alpha \in(0,1]$, then the function

$$
\varphi(\eta):=\alpha \gamma(\alpha ; \eta), \quad \eta \geq 0
$$

is the Laplace exponent of a finite-activity (or step) subordinator $S_{\alpha}:=\left\{S_{\alpha}(t), t \geq 0\right\}$, with triplet $(0,0, \pi)$, where $\pi$ is an absolutely continuous Lévy measure, with completely monotone density

$$
\bar{\pi}(z)=\frac{1_{z \geq 1} \alpha(z-1)^{-\alpha} z^{-1}}{\Gamma(1-\alpha)} .
$$

Proof. The incomplete gamma function $\gamma(\alpha, x)$ is a Bernstein function, as explained in Section 2.3. Hence, also $\alpha \gamma(\alpha, x)$ is a Bernstein function. We now prove that representation (3) holds, in this case, for $a=b=0$ and for the Lévy measure given in (11); indeed, we have that

$$
\begin{aligned}
\int_{0}^{+\infty}\left(1-e^{-\eta x}\right) \pi(d x) & =\int_{0}^{+\infty} x \int_{0}^{\eta} e^{-z x} d z \bar{\pi}(x) d x \\
& =\int_{0}^{\eta} d z \int_{1}^{+\infty} x e^{-z x} \frac{\alpha(x-1)^{-\alpha} x^{-1}}{\Gamma(1-\alpha)} d x \\
& =\int_{0}^{\eta} e^{-z} d z \int_{0}^{+\infty} e^{-z w} \frac{\alpha w^{-\alpha}}{\Gamma(1-\alpha)} d w \\
& =\int_{0}^{\eta} \frac{\alpha e^{-z}}{z^{1-\alpha}} d z=\alpha \gamma(\alpha ; \eta),
\end{aligned}
$$

where the interchange of the integral order is allowed by the absolute convergence of the double integral and the application of the Fubini theorem. In order to prove that $S_{\alpha}$ does not have strictly increasing trajectories, we must show that the integral of the Lévy measure on $(0, \infty)$ is finite. Indeed, by (3) the last condition, together with $a=b=0$, is sufficient to prove that a subordinator is a step process (i.e., it has piecewise sample paths), see [6], p. 135; in this case, we have that

$$
\int_{0}^{+\infty} \pi(d z)=\int_{1}^{+\infty} \frac{\alpha(z-1)^{-\alpha} z^{-1}}{\Gamma(1-\alpha)} d z=\frac{\alpha \Gamma(1-\alpha) \Gamma(\alpha)}{\Gamma(1-\alpha)}=\alpha \Gamma(\alpha)<\infty,
$$

by considering formula (3.191.2) of [15], since $\alpha>0$. Finally, it is easy to check, by differentiating, that the density of the Lévy measure in (11) is completely monotone. 
Remark 1. In the limiting case where $\alpha \rightarrow 1^{-}$the process $S_{\alpha}$ reduces to the Poisson process. We have that $\lim _{\alpha \rightarrow 1^{-}} \pi(d z)=\delta_{1}(z) d z$, which is the Lévy measure of the Poisson process of rate 1 ; this can be seen by considering that

$$
\lim _{\alpha \rightarrow 1^{-}} \varphi(\eta)=\gamma(1, \eta)=1-e^{-\eta}=\int_{0}^{+\infty}\left(1-e^{-\eta x}\right) \delta_{1}(x) d x .
$$

We underline that the Lévy measure given in (11) is different from zero only for $z \geq 1$; this means that the subordinator exhibits almost surely jumps of size greater than one. As a consequence, and by considering that its diffusion coefficient is zero, the process $S_{\alpha}$ has also finite variation (see Theorem 21.9 in [6]).

The result in (12) implies that $S_{\alpha}$ is a Lévy process of type A (see Definition 11.9 in [6], p. 65) and has finite activity, i.e., the number of jumps is finite on every compact interval for almost all the paths (see Theorem 21.3 in [6]). Thus, $S_{\alpha}$ can be represented as a compound Poisson process

$$
S_{\alpha}(t)=\sum_{j=1}^{N_{\alpha}(t)} Z_{j}^{\alpha}
$$

where $N_{\alpha}:=\left\{N_{\alpha}(t), t \geq 0\right\}$ is a homogeneous Poisson process with the rate $\lambda=\alpha \Gamma(\alpha)$ and the jumps $Z_{j}^{\alpha}$ are i.i.d. random variables, taking values in $[1,+\infty)$, with probability density

$$
f_{Z^{\alpha}}(z)=\frac{(z-1)^{-\alpha} z^{-1} 1_{z \geq 1}}{\Gamma(1-\alpha) \Gamma(\alpha)}=\frac{\sin (\pi \alpha)}{\pi} \frac{1_{z \geq 1}}{(z-1)^{\alpha} z}, \quad \alpha \in(0,1) .
$$

For $\alpha=1$, the jumps are unitary, and the process coincides with the standard Poisson. The representation (13) can be checked directly as follows: the Laplace transform of the addends $Z_{j}^{\alpha}$ is given by

$$
\mathbb{E} e^{-\eta Z_{j}^{\alpha}}=\frac{\sin (\pi \alpha)}{\pi} \int_{1}^{+\infty}(z-1)^{-\alpha} z^{-1} e^{-\eta z} d z=\frac{\Gamma(\alpha ; \eta)}{\Gamma(\alpha)}
$$

for any $j=1,2, \ldots$, by formula (3.383.9) in [15] for $\alpha<1$. Then, by conditioning, we obtain

$$
\begin{aligned}
\mathbb{E} e^{-\eta \sum_{j=1}^{N_{\alpha}(t)} Z_{j}^{\alpha}} & =\exp \left\{-\alpha \Gamma(\alpha) t\left[1-\frac{\Gamma(\alpha ; \eta)}{\Gamma(\alpha)}\right]\right\} \\
& =\exp \{-t \alpha \gamma(\alpha ; \eta)\}
\end{aligned}
$$

Finally, we note that $S_{\alpha}$ is not self-similar, as can be checked from its Laplace transform. The moments of any integer order of $S_{\alpha}$ are not finite, for any $t>0$, since

$$
\int_{1}^{+\infty}|x|^{k} \pi(d x)=\int_{1}^{+\infty} \frac{\alpha(x-1)^{-\alpha} x^{k-1}}{\Gamma(1-\alpha)} d x
$$

does not converge, for any $k \geq 1$, (see [10], p. 132). Alternatively, this can be seen by applying the Wald formula and by noting that $\mathbb{E} Z_{j}^{\alpha}=\frac{\sin (\pi \alpha)}{\pi} \int_{1}^{+\infty}(z-1)^{-\alpha} d z=+\infty$, $j=1,2, \ldots$.

The reason can be found in the heaviness of its distribution's tail. It can be proven that it displays the same power law of the stable subordinator, i.e., $P\left(X_{\alpha}(t)>x\right) \simeq \frac{t x^{-\alpha}}{\Gamma(1-\alpha)}$ for large $x$ (see [2], p. 17).

However, we can study the asymptotic expression of the fractional moment of $S_{\alpha}$, of order $p \leq \alpha$ and for large $t$. We recall that the fractional moments have been introduced and studied by many authors in order to overcome the problem of infinite integer order moments, especially in the stable case (see, among the others, [16,17]); in particular, we will follow the techniques given in [18], which are based on fractional differentiation of the Laplace transform. 
Theorem 2. (1) Let $\alpha \in(0,1)$, then, for any $t \geq 0$ and for $x \rightarrow+\infty$, we have that

$$
P\left(S_{\alpha}(t)>x\right) \simeq \frac{t x^{-\alpha}}{\Gamma(1-\alpha)} .
$$

(2) Let $p \in(0,1]$, then the fractional moment of order $p$ of the process $S_{\alpha}$ exists, finite, for $p \leq \alpha$, and it asymptotically behaves as follows

$$
\mathbb{E} S_{\alpha}^{p}(t) \simeq \frac{\Gamma\left(1-\frac{p}{\alpha}\right)}{\Gamma(1-p)} t^{p / \alpha}, \quad t \rightarrow+\infty .
$$

Proof. We can write, for $\eta \rightarrow 0$,

$$
\begin{aligned}
\int_{0}^{+\infty} e^{-\eta x} P\left(S_{\alpha}(t)>x\right) d x & =\frac{1-\mathbb{E} e^{-\eta S_{\alpha}(t)}}{\eta}=\frac{1-e^{-t \alpha \gamma(\alpha ; \eta)}}{\eta} \\
& \simeq t \eta^{\alpha-1}
\end{aligned}
$$

where we have taken the Taylor series expansion (up to the first order), and we have considered the asymptotic behavior of the lower incomplete gamma function, i.e.,

$$
\gamma(\alpha ; \eta) \simeq \frac{\eta^{\alpha}}{\alpha}, \quad \eta \rightarrow 0
$$

Formula (18) can be easily derived by rewriting (1) as follows:

$$
\gamma(\alpha ; x)=x^{\alpha} \int_{0}^{1} e^{-x w} w^{\alpha-1} d w .
$$

By applying the Tauberian theorem (see [19], Thm.4, p. 446) we find, for any $t \geq 0$, result (16).

In order to derive the asymptotic behavior of the fractional moment of order $p$, we apply the Laplace-Erdelyi Theorem to the following integral

$$
\begin{aligned}
\mathbb{E} S_{\alpha}^{p}(t) & =-\frac{1}{\Gamma(1-p)} \int_{0}^{+\infty} \frac{d}{d \eta}\left[e^{-t \alpha \gamma(\alpha ; \eta)}\right] \eta^{-p} d \eta \\
& =\frac{\alpha t}{\Gamma(1-p)} \int_{0}^{+\infty} e^{-\eta-t \alpha \gamma(\alpha ; \eta)} \eta^{\alpha-p-1} d \eta,
\end{aligned}
$$

(see [20], for details). Let $x \in\left(x_{0}, x_{1}\right)$, with $x_{0}, x_{1} \in \mathbb{R}$, let, moreover, $h(x)$ and $\varphi(x)$ be independent of $t>0$ and $h(x)>h\left(x_{0}\right)$ for all $x \in\left(x_{0}, x_{1}\right)$. Let the following expansions hold, for $x \rightarrow x_{0}^{+}, h(x) \sim h\left(x_{0}\right)+\sum_{k=0}^{\infty} a_{k}\left(x-x_{0}\right)^{k+\mu}, \mu \in \mathbb{R}^{+}, a_{0} \neq 0$, and $\varphi(x) \sim$ $\sum_{k=0}^{\infty} b_{k}\left(x-x_{0}\right)^{k+\gamma-1}, \gamma \in \mathbb{R}^{+}, b_{0} \neq 0$. Then,

$$
I(t):=\int_{x_{0}}^{x_{1}} \varphi(x) e^{-t h(x)} d x \sim e^{-t h\left(x_{0}\right)} \sum_{j=0}^{\infty} \frac{c_{j}}{t^{\frac{\gamma+j}{\mu}}} \Gamma\left(\frac{\gamma+j}{\mu}\right), \quad t \rightarrow+\infty,
$$

under the assumption that the integral (with finite or infinite delimiters) converges absolutely for all sufficiently large $t$. We only need $c_{0}=b_{0} / \mu a_{0}^{\gamma / \mu}$, then, for the expressions of the other $c_{j}$ 's, we refer to $[20,21]$. In our case, we have that $\varphi(x):=e^{-x} x^{\alpha-p-1}=$ $\sum_{k=0}^{\infty} \frac{(-1)^{k} x^{k+\alpha-p-1}}{k !}$, so that $\gamma=\alpha-p>0$, for $p<\alpha$, and $b_{0}=1$. On the other hand, we have $h(x):=\alpha \gamma(\alpha ; x)=\alpha \gamma(\alpha ; 0)+\alpha \sum_{k=0}^{\infty} \frac{(-1)^{k} x^{k+\alpha}}{k !(\alpha+k)}$, by using the well-known series expression of the incomplete gamma function (see [22]). Thus, we have $\mu=\alpha$ and $a_{0}=1$. By considering (19) we, thus, obtain 


$$
\mathbb{E} S_{\alpha}^{p}(t) \sim \frac{\alpha t}{\Gamma(1-p)} \sum_{j=0}^{\infty} \frac{c_{j}}{t^{\frac{\alpha-p+j}{\alpha}}} \Gamma\left(\frac{\alpha-p+j}{\alpha}\right) \sim \frac{\alpha c_{0} t^{p / \alpha} \Gamma\left(1-\frac{p}{\alpha}\right)}{\Gamma(1-p)},
$$

which coincides with (17).

Remark 2. The fractional moment of order $p$ converges, for $t \rightarrow+\infty$, to the value obtained in the stable case, for any $t$ (see [23]).

\subsection{Link to Stable Subordinators}

\subsubsection{The One-Dimensional Case}

We now purpose a slight generalization of the previous results, in order to provide an approximation of a stable subordinator: while the previously defined subordinator $S_{\alpha}$ performs jumps greater than 1, we now consider a lower bound for the jump size equal to $\epsilon>0$.

We, thus, define the following Lévy measure with support on $(\epsilon, \infty)$ and with density

$$
\pi_{\epsilon}(x)=\frac{\alpha}{\Gamma(1-\alpha)}(x-\epsilon)^{-\alpha} x^{-1} 1_{x \geq \epsilon} .
$$

The corresponding Laplace exponent has the form

$$
\varphi_{\varepsilon}(\eta)=\frac{\alpha}{\epsilon^{\alpha}} \gamma(\alpha ; \eta \epsilon)
$$

Indeed,

$$
\begin{aligned}
\varphi_{\epsilon}(\eta) & =\int_{0}^{\infty}\left(1-e^{-\eta x}\right) \pi_{\epsilon}(x) d x \\
& =\int_{\epsilon}^{\infty}\left(1-e^{-\eta x}\right) \frac{\alpha}{\Gamma(1-\alpha)}(x-\epsilon)^{-\alpha} x^{-1} d x \\
& =\int_{\epsilon}^{\infty} d x \frac{\alpha}{\Gamma(1-\alpha)}(x-\epsilon)^{-\alpha} x^{-1} \int_{0}^{\eta} x e^{-x z} d z \\
& =\int_{0}^{\eta} d z \int_{0}^{\infty} \frac{\alpha y-\alpha}{\Gamma(1-\alpha)} e^{-z(y+\epsilon)} d y \\
& =\frac{\alpha}{\epsilon^{\alpha}} \int_{0}^{\eta \epsilon} e^{-w} w^{\alpha-1} d w .
\end{aligned}
$$

By a simple change of variable, the Laplace exponent can also be expressed as $\eta^{\alpha}$ multiplied by a correction factor depending on $\epsilon$ :

$$
\varphi_{\epsilon}(\eta)=\eta^{\alpha} \cdot O_{\epsilon}(\eta)
$$

where

$$
O_{\epsilon}(\eta)=\frac{\alpha}{\epsilon^{\alpha}} \int_{0}^{\epsilon} e^{-\eta y} y^{\alpha-1} d y
$$

is such that $O_{\epsilon}(\eta) \rightarrow 1$, as $\epsilon \rightarrow 0$. Thus, in the limit as $\epsilon \rightarrow 0$, the related subordinator $S_{\alpha}^{(\varepsilon)}:=\left\{S_{\alpha}^{(\varepsilon)}(t), t \geq 0\right\}$ converges to a $\alpha$-stable subordinator, since

$$
\begin{aligned}
& \pi_{\epsilon}(x) \rightarrow \frac{\alpha}{\Gamma(1-\alpha)} x^{-\alpha-1} 1_{x \geq 0} \\
& \varphi_{\epsilon}(\eta) \rightarrow \eta^{\alpha}
\end{aligned}
$$

By considering that

$$
\int_{0}^{\infty} \pi_{\epsilon}(x) d x=\alpha \Gamma(\alpha) \epsilon^{-\alpha}
$$


we can conclude that $S_{\alpha}^{(\varepsilon)}$ is a compound Poisson process, i.e.,

$$
S_{\alpha}^{(\varepsilon)}(t)=\sum_{j=1}^{N^{\epsilon}(t)} Z_{j}^{\epsilon}
$$

where $N^{\epsilon}(t)$ is a Poisson process with intensity $\alpha \Gamma(\alpha) \epsilon^{-\alpha}$, and $Z_{j}^{\epsilon}$ has density

$$
f_{Z_{j}^{\varepsilon}}(z)=\frac{\epsilon^{\alpha}}{\Gamma(\alpha) \Gamma(1-\alpha)}(z-\epsilon)^{-\alpha} z^{-1} 1_{z \geq \epsilon}
$$

Thus, $S_{\alpha}^{(\varepsilon)}$ is a compound Poisson approximation of a stable subordinator. Therefore, it can be useful in many applications, since it is easier to be handled with respect to the stable subordinator, due to its finite activity.

As far as the governing equation is concerned, we can show that the transition density of $S_{\alpha}^{(\varepsilon)}$ satisfies a fractional equation, which generalizes the governing Equation (5) of the stable subordinator. In particular, the fractional derivative on the right side is corrected by means of the following operator

$$
\begin{aligned}
O_{\epsilon}\left(\frac{\partial}{\partial x}\right) h(x) & :=\frac{\alpha}{\epsilon^{\alpha}} \int_{0}^{\epsilon} e^{-y \partial x} h(x) y^{\alpha-1} d y \\
& =\frac{\alpha}{\epsilon^{\alpha}} \int_{0}^{\epsilon} h(x-y) y^{\alpha-1} d y,
\end{aligned}
$$

where $h: \mathbb{R}^{+} \rightarrow \mathbb{R}$ is a function such that the above integral converges, while $e^{-y \partial_{x}}$ denotes (with a little abuse of notation), the translation operator.

Note that (22) tends to the identity operator as $\epsilon \rightarrow 0$, since

$$
\lim _{\epsilon \rightarrow 0} O_{\epsilon}\left(\frac{\partial}{\partial x}\right) h(x)=h(x) .
$$

Thus, we can check that the density $q_{\varepsilon}:=q_{\varepsilon}(x, t), x, t \geq 0$, of $S_{\alpha}^{(\varepsilon)}$ solves the following equation

$$
\frac{\partial}{\partial t} q_{\epsilon}(x, t)=-\frac{\partial^{\alpha}}{\partial x^{\alpha}} O_{\epsilon}\left(\frac{\partial}{\partial x}\right) q_{\epsilon}(x, t) \quad q_{\epsilon}(x, 0)=\delta(x),
$$

by applying the Laplace transform to both members, which gives

$$
\widetilde{q}_{\epsilon}(\eta, t)=e^{-\eta^{\alpha} O_{\epsilon}(\eta) t}
$$

where $O_{\epsilon}(\eta)$ has been defined in (21).

Remark 3. The approximation presented above could be applied to the fractional derivative with time-dependent order, i.e., $\left(\frac{\partial}{\partial x}\right)^{\alpha(t)}$, where $\alpha(t)$ takes values in $(0,1)$. Such an operator governs a time-inhomogeneous version of the stable subordinator (see, for example, $[24,25]$ ), which could be approximated by considering the time-dependent Lévy measure $\pi_{\epsilon}(x, t)=\frac{\alpha(t)}{\Gamma(1-\alpha(t))}(x-$ $\epsilon)^{-\alpha(t)} x^{-1} 1_{x \geq \epsilon}$.

\subsubsection{The Multivariate Case}

Following the lines of the one-dimensional case, we look for a compound Poisson approximation for a multivariate stable subordinator, which we introduced in Section 2.2. We define the family of Lévy measures

$$
v_{\epsilon}(d \rho, d \theta)=C(\rho-\epsilon)^{-\alpha} \rho^{-1} M(d \theta) \quad \epsilon>0
$$


and, by the same calculations as in (20), we obtain the following family of Bernstein functions (the symbol $\eta$ denotes the vector $\left(\eta_{1}, \ldots, \eta_{d}\right)$ and $\cdot$ denotes the scalar product)

$$
\begin{aligned}
\Phi_{\epsilon}(\eta) & =k \int_{0}^{\infty} d \rho \int_{B_{+}^{d-1}}\left(1-e^{-\rho \eta \cdot \theta}\right)(\rho-\epsilon)^{-\alpha} \rho^{-1} M(d \theta) \\
& =k \int_{B_{+}^{d-1}}(\theta \cdot \eta)^{\alpha} O_{\epsilon}(\eta \cdot \theta) M(d \theta)
\end{aligned}
$$

where the corrective term

$$
O_{\epsilon}(\eta \cdot \theta)=\frac{\alpha}{\epsilon^{\alpha}} \int_{0}^{\epsilon} e^{-\eta \cdot \theta y} y^{\alpha-1} d y
$$

tends to 1 as $\epsilon \rightarrow 0$. By Laplace inversion, the density $q_{\epsilon}(x, t)$ of our process satisfies

$$
\frac{\partial}{\partial t} q_{\epsilon}(x, t)=-k \int_{B_{+}^{d-1}}\left(\nabla_{x} \cdot \theta\right)^{\alpha} O_{\epsilon}\left(\theta \cdot \nabla_{x}\right) q_{\epsilon}(x, t) M(d \theta)
$$

where

$$
\begin{aligned}
O_{\epsilon}\left(\theta \cdot \nabla_{x}\right) h(x):= & \frac{\alpha}{\epsilon^{\alpha}} \int_{0}^{\epsilon} e^{-y \theta \cdot \nabla_{x}} h(x) y^{\alpha-1} d y \\
& =\frac{\alpha}{\epsilon^{\alpha}} \int_{0}^{\epsilon} h(x-y \theta) y^{\alpha-1} d y
\end{aligned}
$$

tends to the identity operator in the limit $\epsilon \rightarrow 0$.

\section{The Tempered Subordinator $S_{\alpha, \theta}$}

In order to avoid the inconvenience of infinite moments of $S_{\alpha}$, we define a tempered counterpart of the latter.

Theorem 3. Let $\eta, \theta>0$ and $\alpha \in(0,1]$, then the function

$$
\varphi_{\theta}(\eta):=\alpha \gamma(\alpha ; \eta+\theta)-\alpha \gamma(\alpha ; \theta),
$$

is the Laplace exponent of a tempered subordinator $S_{\alpha, \theta}:=\left\{S_{\alpha, \theta}(t), t \geq 0\right\}$, with Léry triplet $\left(0,0, \pi_{\theta}\right)$ and (absolutely continuous) Léry measure $\pi_{\theta}$, with density

$$
\bar{\pi}_{\theta}(z)=\frac{1_{z \geq 1} \alpha(z-1)^{-\alpha} z^{-1} e^{-\theta z}}{\Gamma(1-\alpha)} .
$$

The sample paths of $S_{\alpha, \theta}$ are not strictly increasing; the mean and variance of $S_{\alpha, \theta}$ read, respectively,

$$
\begin{aligned}
\mathbb{E} S_{\alpha, \theta}(t) & =t \alpha \theta^{\alpha-1} e^{-\theta} \\
\operatorname{Var} S_{\alpha, \theta}(t) & =t \alpha \theta^{\alpha-1} e^{-\theta}+\alpha(1-\alpha) t \theta^{\alpha-2} e^{-\theta} .
\end{aligned}
$$

Proof. It is immediate to check that (23) is a Bernstein function (as a consequence of Theorem 1). We can prove that the representation (3) holds, in this case, for $a=b=0$ and for the Lévy density given in (24); indeed, we have that 


$$
\begin{aligned}
\int_{0}^{+\infty}\left(1-e^{-\eta x}\right) \bar{\pi}_{\theta}(x) d x & =\int_{0}^{+\infty} x \int_{0}^{\eta} e^{-z x} d z \bar{\pi}_{\theta}(x) d x \\
& =\int_{0}^{\eta} d z \int_{1}^{+\infty} x e^{-z x} \frac{\alpha(x-1)^{-\alpha} x^{-1} e^{-\theta x}}{\Gamma(1-\alpha)} d x \\
& =\int_{0}^{\eta} e^{-(z+\theta)} d z \int_{0}^{+\infty} e^{-(z+\theta) w} \frac{\alpha w^{-\alpha}}{\Gamma(1-\alpha)} d w \\
& =\alpha \int_{\theta}^{\theta+\eta} e^{-w} w^{\alpha-1} d z,
\end{aligned}
$$

which coincides with (23). In this case, the Lévy measure is finite, since

$$
\int_{0}^{+\infty} \pi_{\theta}(d z)=\int_{1}^{+\infty} \frac{\alpha(z-1)^{-\alpha} z^{-1} e^{-\theta z}}{\Gamma(1-\alpha)} d z=\alpha \Gamma(\alpha ; \theta)<\infty,
$$

by considering (14). The mean and variance given in (25) can be obtained by differentiating the Laplace transform

$$
\mathbb{E} e^{-\eta S_{\alpha, \theta}(t)}=e^{-t \alpha \int_{\theta}^{\theta+\eta} e^{-w} w^{\alpha-1} d z},
$$

with respect to $\eta$ and considering the relationship $\mathbb{E}\left[S_{\alpha, \theta}(t)\right]^{k}=\left.(-1)^{k} \frac{\partial^{k}}{\partial \eta^{k}} \mathbb{E} e^{-\eta S_{\alpha, \theta}(t)}\right|_{\eta=0^{\prime}}$ for $k \in \mathbb{N}$.

Remark 4. It is easy to check that the mean and variance of $S_{\alpha, \theta}$, given in (25), tend to infinity, as $\theta \rightarrow 0$, as expected from (15).

Remark 5. From (26), we can infer that the process $S_{\alpha, \theta}$ has finite activity and that the following compound Poisson representation holds

$$
S_{\alpha, \theta}(t)=\sum_{j=1}^{N_{\alpha, \theta}(t)} Z_{j}^{\alpha, \theta},
$$

where $N_{\alpha, \theta}:=\left\{N_{\alpha, \theta}(t), t \geq 0\right\}$ is a homogeneous Poisson process with rate $\lambda=\alpha \Gamma(\alpha ; \theta)$. The jumps $Z_{j}^{\alpha, \theta}$ are i.i.d. random variables, taking values in $[1,+\infty)$ and with the probability density function

$$
f_{Z^{\alpha, \theta}}(z)=\frac{1_{z \geq 1}(z-1)^{-\alpha} z^{-1} e^{-\theta z}}{\Gamma(1-\alpha) \Gamma(\alpha ; \theta)}, \quad \alpha \in(0,1) .
$$

For $\alpha=1$, Formula (23) reduces to $\varphi_{\theta}(\eta)=\gamma(1 ; \eta+\theta)-\gamma(1 ; \theta)=e^{-\vartheta}\left(e^{-\eta}-1\right)$, which is the Laplace exponent of a Poisson process of rate $e^{-\theta}$. This is confirmed by its Léry measure, which is obtained from (24), since $\lim _{\alpha \rightarrow 1} \pi_{\theta}(d z)=e^{-\theta} \delta_{1}(z) d z$. Indeed, the process $N_{1, \theta}$ in (28) has rate $\lambda=\Gamma(1 ; \theta)=e^{-\vartheta}$, in this special case.

\section{Subordination of Lévy Processes}

We now consider the subordination of a Lévy process $X(t)$ by means of $\beta_{0} t+S_{\alpha, \theta}(t)$, where $S_{\alpha, \theta}$ is the tempered subordinator defined above and $\beta_{0} \geq 0$ is a possible drift parameter. Let $(a, b, v)$ be the Lévy triplet of $X$ and $\mu$ be its probability distribution, i.e., $\mu_{t}(B):=P(X(t) \in B)$, for any Borel set $B$. We assume that $X$ is independent of $S_{\alpha, \theta}$.

Then, by applying Thm. 30.1, p. 197 in [6], the process $Z:=\{Z(t), t \geq 0\}$ defined as

$$
Z(t):=X\left(\beta_{0} t+S_{\alpha, \theta}(t)\right), \quad t \geq 0,
$$

is a Lévy process with triplet $\left(a^{\prime}, b^{\prime}, v^{\prime}\right)$, where 


$$
\begin{aligned}
a^{\prime} & =\beta_{0} a \\
b^{\prime} & =\beta_{0} b+\int_{0}^{+\infty} \pi_{\theta}(d z) \int_{|x| \leq 1} x \mu_{z}(d x), \\
v^{\prime}(d x) & =\beta_{0} v(d x)+\int_{1}^{+\infty} \mu_{z}(d x) \pi_{\theta}(d z) .
\end{aligned}
$$

By considering Prop.1.3.27 in [10], we can also derive the Lévy symbol of the subordinated process, which is again expressed in terms of incomplete gamma functions:

$$
\psi_{Z}(u)=-\varphi_{\theta}\left(-\psi_{X}(u)\right)=\alpha \gamma(\alpha ; \theta)-\alpha \gamma\left(\alpha ; \theta-\psi_{X}(u)\right) .
$$

\subsection{The Generator Equation}

Let us consider the case $\beta_{0}=\theta=0$. For $h \in B_{b}(\mathbb{R})$, where $B_{b}(\mathbb{R})$ denotes the set of real-valued bounded Borel measurable functions, equipped with the sup-norm. The operator $T_{t}$ defined by

$$
T_{t} h(x)=\mathbb{E} h(x+X(t))
$$

defines a strongly continuous contraction semigroup on $B_{b}(\mathbb{R})$. If $A$ is the generator of $T_{t}$, then (32) satisfies

$$
\frac{\partial}{\partial t} g(x, t)=A g(x, t) \quad g(x, 0)=h(x)
$$

for $h$ in the domain of $A$. If $\sigma_{\alpha}(t)$ is a stable subordinator, then the process $X\left(\sigma_{\alpha}(t)\right)$ induces the subordinate semigroup

$$
\tilde{T}_{t} h(x)=\mathbb{E} h\left(x+X\left(\sigma_{\alpha}(t)\right)\right) .
$$

In light of the Phillips theorem (see [6], page 212), the semigroup (33) satisfies

$$
\frac{\partial}{\partial t} g(x, t)=-(-A)^{\alpha} g(x, t), \quad g(x, 0)=h(x),
$$

where the fractional power of the operator is defined by

$$
-(-A)^{\alpha} h(x)=\int_{0}^{\infty}\left(T_{s} h(x)-h(x)\right) \frac{\alpha}{\Gamma(1-\alpha)} s^{-\alpha-1} d s
$$

at least on the same domain of $A$.

Now, if we employ the subordinator $S_{\alpha}^{(\epsilon)}$, which is an approximation of $\sigma_{\alpha}$ (see the discussion in Section 3.2.1), we obtain an approximation of Equation (34). Indeed, using again the Phillips theorem,

$$
T_{t}^{\epsilon} h(x)=\mathbb{E} h\left(x+X\left(S_{\alpha}^{\epsilon}(t)\right)\right.
$$

satisfies the following equation

$$
\frac{\partial}{\partial t} g(x, t)=\int_{\epsilon}^{\infty}\left(T_{s} g(x, t)-g(x, t)\right) \frac{\alpha(s-\epsilon)^{-\alpha} s^{-1}}{\Gamma(1-\alpha)} d s, \quad g(x, 0)=h(x) .
$$

The operator on the right-side is an approximation of the fractional power in (35), to which it converges as $\epsilon \rightarrow 0$.

We observe that, in the special case $X(t)=t$, i.e., when $T_{t}$ is the shift operator, the operator on the right-side is an approximation of the Marchaud fractional derivative, namely

$$
\int_{\epsilon}^{\infty}(g(x-s, t)-g(x, t)) \frac{\alpha(s-\epsilon)^{-\alpha} s^{-1}}{\Gamma(1-\alpha)} d s .
$$




\subsection{Subordinated Brownian Motion}

In the Brownian case, i.e., when the external process $X$ is a standard Brownian motion $B:=\{B(t), t \geq 0\}$ and the triplet is $(0,1,0)$, we have, from (30), that the Lévy process

$$
Z(t)=B\left(\beta_{0} t+S_{\alpha, \theta}(t)\right)
$$

is given by the superposition of a Brownian motion (with diffusion coefficient $\beta_{0}$ ) and a jump process. Indeed it has the Lévy triplet $\left(0, \beta_{0}, v^{\prime}\right)$, where

$$
\begin{aligned}
v^{\prime}(x) & =\int_{1}^{+\infty} \frac{e^{-\frac{x^{2}}{2 z}}}{\sqrt{2 \pi z}} \frac{\alpha(z-1)^{-\alpha} z^{-1} e^{-\theta z}}{\Gamma(1-\alpha)} d z \\
& =\frac{\alpha}{\Gamma(1-\alpha) \sqrt{2 \pi}} \sum_{j=0}^{+\infty} \frac{\left(-x^{2} / 2\right)^{j}}{j !} \int_{1}^{+\infty}(z-1)^{1-\alpha-1} z^{-j-\frac{1}{2}-1} e^{-\theta z} d z \\
& =\frac{\alpha e^{-\theta / 2} \theta^{\frac{\alpha}{2}-\frac{1}{4}}}{\sqrt{2 \pi}} \sum_{j=0}^{+\infty} \frac{\left(-x^{2} \sqrt{\theta} / 2\right)^{j}}{j !} W_{\frac{\alpha}{2}-\frac{j}{2}-\frac{3}{4}, \frac{\alpha}{2}+\frac{j}{2}+\frac{1}{4}}(\theta(1-\alpha)),
\end{aligned}
$$

by (3.383.4) in [15], where $W_{\beta, \gamma}(\cdot)$ denotes the Whittaker function (see also [16], p. 27), by considering that $1-\alpha>0$ and $\theta>0$. In the special case where $\theta=0$, i.e., in the non-tempered case, we have, instead, the following easier expression

$$
\begin{aligned}
v^{\prime}(x) & =\int_{1}^{+\infty} \frac{e^{-\frac{x^{2}}{2 z}}}{\sqrt{2 \pi z}} \frac{\alpha(z-1)^{-\alpha} z^{-1}}{\Gamma(1-\alpha)} d z \\
& =\int_{0}^{1} \frac{e^{-\frac{x^{2} w}{2}}}{\sqrt{2 \pi}} \frac{\alpha w^{\alpha+\frac{1}{2}-1}(1-w)^{1-\alpha-1}}{\Gamma(1-\alpha)} d w \\
& =\frac{\sqrt{2} \alpha \Gamma\left(\alpha+\frac{1}{2}\right)}{\pi}{ }_{1} F_{1}\left(\alpha+\frac{1}{2} ; \frac{3}{2} ;-\frac{x^{2}}{2}\right),
\end{aligned}
$$

by (1.6.15) in [12] [a $\left.=\alpha+\frac{1}{2}, c=\frac{3}{2}\right]$, where ${ }_{1} F_{1}(a ; c ; z)=\sum_{k=0}^{\infty} \frac{(a)_{k}}{(c)_{k}} \frac{z^{k}}{k !}$ is the confluent hypergeometric Kummer function, which is defined for any $a, z \in \mathbb{C}$ and $c \in \mathbb{C} \backslash \mathbb{Z}_{0}^{-}$ (see [12], p. 29, for details). Due to formula (1.9.3) in [12], p. 45, we can write (36) in terms of the generalized (three-parameters) Mittag-Leffler function, as follows

$$
v^{\prime}(x)=\alpha \frac{\Gamma\left(\alpha+\frac{1}{2}\right)}{\sqrt{2 \pi}} E_{1,3 / 2}^{\alpha+1 / 2}\left(-\frac{x^{2}}{2}\right),
$$

where $E_{\alpha, \beta}^{\gamma}(z):=\sum_{k=0}^{\infty} \frac{(\gamma)_{k} z^{k}}{k ! \Gamma(\alpha k+\beta)}$ and $(\gamma)_{k}:=\gamma(\gamma+1) \ldots(\gamma+n-1)$, for $z, \alpha, \beta, \gamma \in \mathbb{C}$ with $\operatorname{Re}(\alpha)>0, n \in \mathbb{N}$.

It is easy to check that the jump component of the subordinated process has finite activity for any $\alpha \in(0,1)$, since

$$
\begin{aligned}
\int_{0}^{+\infty} v^{\prime}(d x) & =\int_{1}^{+\infty} \frac{\alpha(z-1)^{-\alpha} z^{-1} e^{-\theta z}}{\Gamma(1-\alpha)} d z \\
& =\alpha \Gamma(\alpha ; \theta)<\infty .
\end{aligned}
$$


By (26), we have that

$$
\begin{aligned}
\int_{|x| \geq 1}|x|^{k} v^{\prime}(d x) & =\int_{1}^{+\infty}\left(\int_{|x| \geq 1}|x|^{k} \frac{e^{-\frac{x^{2}}{2 z}}}{\sqrt{2 \pi z}} d x\right) \frac{\alpha(z-1)^{-\alpha} z^{-1} e^{-\theta z}}{\Gamma(1-\alpha)} d z \\
& \leq \int_{1}^{+\infty} \mathbb{E}|B(z)|^{k} \frac{\alpha(z-1)^{-\alpha} z^{-1} e^{-\theta z}}{\Gamma(1-\alpha)} d z \\
& =\frac{2^{k / 2} \Gamma\left(\frac{k+1}{2}\right)}{\sqrt{\pi}} \int_{1}^{+\infty} \frac{\alpha(z-1)^{1-\alpha-1} z^{\frac{k}{2}-1} e^{-\theta z}}{\Gamma(1-\alpha)} d z .
\end{aligned}
$$

The characteristic function of $Z(t)$ is given by

$$
\mathbb{E} e^{i u B\left(\beta_{0} t+S_{\alpha, \theta}(t)\right)}=\exp \left\{-\frac{1}{2} u^{2} \beta_{0} t-t \alpha \int_{\theta}^{\theta+u^{2} / 2} e^{-w} w^{\alpha-1} d w\right\} .
$$

By conditioning and considering (25), we have that $\mathbb{E} B\left(\beta_{0} t+S_{\alpha, \theta}(t)\right)=0$, for any $t, \theta \geq 0$, and the autocovariance of the subordinated Brownian motion, for any $t, \tau \geq 0$, reads

$$
\begin{aligned}
\operatorname{Cov}\left(B\left(\beta_{0} t+S_{\alpha, \theta}(t)\right), B\left(\beta_{0} \tau+S_{\alpha, \theta}(\tau)\right)\right) & =\mathbb{E}\left(\left(\beta_{0} t+S_{\alpha, \theta}(t)\right) \wedge\left(\beta_{0} \tau+S_{\alpha, \theta}(\tau)\right)\right. \\
& =\mathbb{E}\left(\beta_{0}(t \wedge \tau)+S_{\alpha, \theta}(t \wedge \tau)\right) \\
& =\beta_{0}(t \wedge \tau)+(t \wedge \tau) \alpha \theta^{\alpha-1} e^{-\theta} .
\end{aligned}
$$

Thus, even if the autocovariance is linear w.r.t. the time argument, the parameters $\alpha$ and $\theta$ can be interpreted as a measure of deviation from the standard Brownian dependence structure: in particular, for $\theta \rightarrow 0$ and for $\alpha$ strictly less than 1 , the autocovariance tends to infinity, for any $t$.

\section{Subordinated Fractional Brownian Motion}

We now consider the process $\left\{B_{H}\left(S_{\alpha}(t)\right), t \geq 0\right\}$, where $B_{H}:=\left\{B_{H}(t), t \geq 0\right\}$ is the fractional Brownian motion (hereafter FBM) with the Hurst parameter $H$ and the subordinator $S_{\alpha}$ is supposed to be independent of it. The FBM $B_{H}$ is defined, for any $H \in(0,1)$ as a self-similar process with index $H$ and with zero-mean Gaussian distribution. Its one dimensional distribution has density

$$
f_{B_{H}}(x, t)=\frac{1}{\sqrt{2 \pi} t^{H}} \exp \left\{-\frac{x^{2}}{2 t^{2 H}}\right\}, \quad x \in \mathbb{R}, t \geq 0 .
$$

It can be expressed, in terms of the standard Brownian motion $B:=\{B(t), t \geq 0\}$, by the following representation

$$
B_{H}(t)=\int_{\mathbb{R}}\left[(t-u)_{+}^{H-1 / 2}-(-u)_{+}^{H-1 / 2}\right] d B(u), \quad t \geq 0
$$

where $x_{+}=\max (x, 0)$.

For details on the fractional Brownian motion we refer to [7]. It is worth recalling that the FBM exhibits subdiffusive dynamics for $H<1 / 2$ and a superdiffusive one for $H>1 / 2$; indeed the moment of order $q$ of the FBM is given by

$$
\mathbb{E}\left|B_{H}(t)\right|^{q}=t^{q H} \mathbb{E}\left|B_{H}(1)\right|^{q}=\sqrt{\frac{2^{q}}{\pi}} \Gamma\left(\frac{q+1}{2}\right) t^{q H} .
$$

(See, for example, [18]).

Different forms of time-changed FBM have been introduced and studied (see $[18,26,27])$.

We prove here that the FBM, subordinated by an independent $S_{\alpha}$, displays the longrange dependence (LRD) property, for $H \in(0,1 / 2)$; moreover, this behavior depends on 
$\alpha$, instead of what happens in the cases of the FBM subordinated by the tempered stable subordinator (studied in [18]) and by the gamma process (analyzed in [26]). Indeed, in the last cases, the LRD rate depends only on the Hurst parameter $H$.

Since the process is not stationary, we use the following definition of long-range dependence: a process $Z(t)$ is said to have the LRD property if, for $s>0$ and $t>s$,

$$
\operatorname{Corr}(Z(t), Z(s)) \sim c(s) t^{-d}, \quad t \rightarrow+\infty,
$$

where $c(s)$ is a constant depending on $s$ and $d \in(0,1)$ (see [28]).

Theorem 4. Let $H \in(0,1 / 2)$ and $\alpha \geq 2 H$. Let

$$
Z_{H}(t):=B_{H}\left(S_{\alpha}(t)\right), \quad t \geq 0,
$$

where $B_{H}$ is the FBM, with Hurst parameter $H$, and $S_{\alpha}$ is supposed to be independent of it. Then, $Z_{H}$ has the $L R D$ behavior given in (40), with $d=1-\frac{H}{\alpha}$.

Proof. We notice that the subordinator, being a compound Poisson process has stationary and independent increments. By conditioning and considering (39), we find, for $q<\alpha / H$,

$$
\begin{aligned}
\mathbb{E}\left|Z_{H}(t)\right|^{q} & =\mathbb{E}\left|B_{H}(1)\right|^{q} \mathbb{E}\left(S_{\alpha}(t)\right)^{q H}=\sqrt{\frac{2^{q}}{\pi}} \Gamma\left(\frac{q+1}{2}\right) \mathbb{E}\left(S_{\alpha}(t)\right)^{q H} \\
& \simeq \sqrt{\frac{2^{q}}{\pi}} \Gamma\left(\frac{q+1}{2}\right) \frac{\Gamma\left(1-\frac{q H}{\alpha}\right)}{\Gamma(1-q H)} t^{q H / \alpha}, \quad t \rightarrow+\infty .
\end{aligned}
$$

By (17), we, thus, evaluate the covariance of the process $Z_{H}$, as follows, for $s<t$,

$$
\begin{aligned}
\mathbb{E}\left(Z_{H}(t) \cdot Z_{H}(s)\right) & =\frac{1}{2}\left\{\mathbb{E}\left(Z_{H}(t)\right)^{2}+\mathbb{E}\left(Z_{H}(s)\right)^{2}-\mathbb{E}\left[Z_{H}(t)-Z_{H}(s)\right]^{2}\right\} \\
& =\frac{1}{2} \mathbb{E}\left(B_{H}(1)\right)^{2}\left\{\mathbb{E}\left(S_{\alpha}(t)\right)^{2 H}+\mathbb{E}\left(S_{\alpha}(s)\right)^{2 H}-\mathbb{E}\left(S_{\alpha}(t-s)\right)^{2 H}\right\} \\
& =[\operatorname{by}(17)] \\
& \sim \frac{1}{2}\left\{\frac{\Gamma\left(1-\frac{2 H}{\alpha}\right)}{\Gamma(1-2 H)} t^{2 H / \alpha}+\mathbb{E}\left(S_{\alpha}(s)\right)^{2 H}-\frac{\Gamma\left(1-\frac{2 H}{\alpha}\right)}{\Gamma(1-2 H)}(t-s)^{2 H / \alpha}\right\} \\
& =\frac{1}{2} \frac{\Gamma\left(1-\frac{2 H}{\alpha}\right)}{\Gamma(1-2 H)} t^{2 H / \alpha}\left\{\frac{2 H}{\alpha} \frac{s}{t}+\mathbb{E}\left(S_{\alpha}(s)\right)^{2 H} \frac{\Gamma(1-2 H)}{\Gamma\left(1-\frac{2 H}{\alpha}\right)} t^{-2 H / \alpha}+O\left(t^{-2}\right)\right\} .
\end{aligned}
$$

By putting $K_{2 H, \alpha}:=\Gamma\left(1-\frac{2 H}{\alpha}\right) / \Gamma(1-2 H)$, we can write $\mathbb{E}\left(Z_{H}(t) \cdot Z_{H}(s)\right) \sim$ $\frac{H}{\alpha} K_{2 H, \alpha} s t^{\frac{2 H}{\alpha}-1}$. Therefore, the correlation function asymptotically behaves as follows, for $t \rightarrow+\infty$,

$$
\operatorname{Corr}\left(Z_{H}(t), Z_{H}(s)\right) \sim \frac{s t^{\frac{2 H}{\alpha}-1}}{\sqrt{t^{\frac{2 H}{\alpha}} s^{\frac{2 H}{\alpha}}}}=s^{1-\frac{H}{\alpha}} t^{-\left(1-\frac{H}{\alpha}\right)} .
$$

Note that we have applied (42) for $q=2$, and thus (43) holds for $\alpha \geq 2 H$, by Theorem 3; as a consequence, the result is limited to the case of a FBM with $H<1 / 2$.

Remark 6. We underline that the values of $H \geq 1 / 2$ are excluded, since, in this range, the $\mathbb{E}\left(S_{\alpha}(t)^{2 H}\right)$ is infinite. To overcome this limitation, we could have used the tempered subordinator $S_{\alpha, \theta}(t)$ (as done in [18], in the stable case); unfortunately, in the tempered case, the function $h(x)$ in (19) would be given by $h(x)=\alpha \gamma(\alpha ; x+\theta)-\alpha \gamma(\alpha ; \theta)$, which cannot be expanded, as requested by the LaplaceErdelyi Theorem. 
Remark 7. We stress that the LRD parameter $d$ is dependent on $\alpha$, on the contrary of what happens in the case of a FBM subordinated by a tempered stable subordinator or by the gamma process, where the rate $d$ of the LRD depends only on the Hurst parameter $H$ and coincides with that of the fractional Brownian motion itself (see [18,26], respectively).

It is evident by (42) that $\operatorname{var}\left(Z_{H}(t)\right) \simeq K t^{2 H / \alpha}$, for $t \rightarrow+\infty$ (where $K$ is a constant depending on $\alpha, H)$, and therefore the process $Z_{H}$ behaves asymptotically as a subdiffusion, according to the parameter $\alpha$. Indeed, $2 H / \alpha$ is always less than one (since, by assumption, $2 H \leq \alpha$ ), and the subdiffusive behavior is more marked the greater the value of $\alpha$ (for any fixed $H)$.

Author Contributions: L.B. and C.R. contributed equally to the conceptualization and the formal analysis of the problem discussed in the paper. Both authors have read and agreed to the published version of the manuscript.

Funding: This research received no external funding.

Institutional Review Board Statement: Not applicable.

Informed Consent Statement: Not applicable.

Data Availability Statement: Not applicable.

Conflicts of Interest: The authors declare no conflict of interest.

\section{References}

1. Beghin, L.; Gajda, J. Tempered relaxation equation and related generalized stable processes. Fract. Calc. Appl. Anal. 2020, 23, 1248. [CrossRef]

2. Samorodnitsky, G.; Taqqu, M. Stable Non-Gaussian Random Processes: Stochastic Models with Infinite Variance; Chapman and All: New York, NY, USA, 1994.

3. Meerschaert, M.; Sikorskii, A. Stochastic Models for Fractional Calculus; De Gruyter Studies in Mathematics Series; Hubert and Co: Berlin, Germany, 2012; Volume 43.

4. Beghin, L.; Macci, C.; Ricciuti, C. Random time-change with inverses of multivariate subordinators: Governing equations and fractional dynamics. Stoch. Process. Their Appl. 2020, 130, 6364-6387. [CrossRef]

5. Barndorff-Nielsen, O.; Pedersen, J.; Sato, K. Multivariate subordination, self-decomposability and stability. Adv. Appl. Probab. 2001, 33, 160-187. [CrossRef]

6. Sato, K.I. Lévy Processes and Infinitely Divisible Distributions; Cambridge Studies in Advanced Aathematics. 68; Cambridge University Press: Cambridge, UK, 1999.

7. Mandelbrot, B.B.; Ness, J.W.V. Fractional Brownian motions, fractional noises and applications. SIAM Rev. 1968, $10,422-437$. [CrossRef]

8. Metzler, J.R.; Klafter, J. The random walk's guide to anomalous diffusion: A fractional dynamics approach. Phys. Rep. 2000, 339, 1-77. [CrossRef]

9. Schilling, R.L.; Song, R.; Vondracek, Z. Bernstein Functions: Theory and Applications; De Gruyter Studies in Mathematics Series; Hubert and Co: Berlin, Germany, 2010; Volume 37.

10. Applebaum, D. Léry Processes and Stochastic Calculus, 2nd ed.; Cambridge University Press: Cambridge, UK, 2009.

11. Bertoin, J. Subordinators: Examples and Applications. In Lectures on Probability Theory and Statistics (Saint-Flour, 1997); Lecture Notes in Mathematics, Volume 1717; Springer: Berlin, Germany, 1999; pp. 1-91.

12. Kilbas, A.A.; Srivastava, H.M.; Trujillo, J.J. Theory and Applications of Fractional Differential Equations, Volume 204 of North-Holland Mathematics Studies; Elsevier Science B.V.: Amsterdam, The Netherlands, 2006.

13. D'Ovidio, M.; Garra, R. Multidimensional fractional advection-dispersion equations and related stochastic processes. Electron. J. Probab. 2014, 19 , 1-31. [CrossRef]

14. Kumar, A.; Vellaisamy, P. Inverse tempered stable subordinators. Stat. Prob. Lett. 2015, 103, 134-141. [CrossRef]

15. Gradshteyn, I.S.; Ryzhik, I.M. Tables of Integrals, Series and Products, 5th ed.; Academic Press: London, UK, 1994.

16. Mathai, A.M.; Saxena, R.K.; Haubold, H.J. The H-Functions: Theory and Applications; Springer: New York, NY, USA, 2010.

17. Wolfe, S.J. On moments of probability distribution functions. In Fractional Calculus and Its Applications; Lecture Notes in Mathematics, Volume 457; Ross, B., Ed.; Springer: Berlin, Germany, 1975; pp. 306-316.

18. Kumar, A.; Gajda, J.; Wyłomanska, A.; Połoczanski, R. Fractional Brownian motion delayed by tempered and inverse tempered stable subordinators. Methodol. Comput. Appl. Probab. 2019, 21, 185-202. [CrossRef]

19. Feller, W. An Introduction Probability Theory and Its Applications, 2nd ed.; Wiley: New York, NY, USA, 1971 ; Volume 2.

20. Wojdylo, J. On the coefficients that arise from Laplace's method. J. Comput. Appl. Math. 2006, 196, 241-266. [CrossRef]

21. Wong, R. Asymptotic Approximations of Integrals; SIAM, Ed.; Academic Press: Philadelphia, PA, USA, 200 
22. Jameson, G.J. The incomplete gamma functions. Math. Gaz. 2016, 100, 298-306. [CrossRef]

23. Matsui, M.; Pawlas, Z. Fractional absolute moments of heavy tailed distributions. Braz. J. Probab. Stat. 2016, 30, $272-298$. [CrossRef]

24. Beghin, L.; Ricciuti, C. Additive geometric stable processes and related pseudo-differential operators. Markov Process. Relat. Fields 2019, 25, 415-444.

25. Orsingher, E.; Ricciuti, C.; Toaldo, B. Time-inhomogeneous jump processes and variable order operators. Potential Anal. 2016, 45, 435-461. [CrossRef]

26. Kumar, A.; Wyłomanska, A.; Połoczanski, R.; Sundar, S. Fractional Brownian motion time-changed by gamma and inverse gamma process. Phys. A Stat. Mech. Its Appl. 2017, 468, 648-667. [CrossRef]

27. Mijena, J.B. Correlation structure of time-changed fractional Brownian motion. 2014, arXiv:1408.4502v1, 1-15 .

28. D'Ovidio, M.; Nane, E. Time dependent random fields on spherical non-homogeneous surfaces. Stoch. Process. Appl. 2014, 124, 2098-2131. [CrossRef] 